\title{
Genetic Variability, Correlation and Path Analysis of F5 Generation of Ridge Gourd (Luffa acutangula (L.) Roxb.) for Yield and Quality
}

\author{
A. Kannan and C. Rajamanickam* \\ Department of Horticulture, Agricultural College and Research Institute, \\ Madurai - 625 104, Tamil Nadu, India \\ *Corresponding author
}

\section{A B S T R A C T}

\begin{tabular}{|l|}
\hline K e y w o r d s \\
Ridge gourd, GCV, \\
PCV, Heritability, \\
Correlation, Path \\
analysis
\end{tabular}

\section{Introduction}

Ridge gourd (Luffa acutangula (L.) Roxb.) is one of the important cucurbitaceous vegetable and belongs o the family cucurbitaceae. Ridge gourd has been cultivated for centuries in tropical, sub-tropical and milder portions of temperate zones. It is popularly known as
The present investigation on genetic variability, correlation and path analysis of $F_{5}$ generations of ridge gourd was carried out at College Orchard, Department of Horticulture, Agricultural College and Research Institute, Madurai, Tamil Nadu during 2018 - 2019 to develop medium size, high yielding and good quality variety suitable for Madurai condition was aimed at. The selected genotypes from the two crosses viz., Virudhunagar local x Periyakottai local $\left(\mathrm{L}_{3} \mathrm{xT}_{1}\right)$ and Virudhunagar local $\mathrm{x}$ Alathur local $\left(\mathrm{L}_{3} \mathrm{xT}_{2}\right)$ of ridge gourd along with their parents were evaluated. This study was laid out in a randomized block design (RBD) with two replications. The results revealed that both the crosses showed low PCV, GCV coupled with high heritability and high genetic advance for the character node to first male flower. Whereas low PCV, GCV coupled with high heritability and low genetic advance for the characters vine length, days to first female flower and sex ratio. Regarding correlation studies revealed that cross $\mathrm{L}_{3} \mathrm{xT}_{1}$ the trait fruit yield was found to be significantly and positively correlated with node to first male flower, node to first female flower, days to first harvest, fruit weight, number of fruits per plant and flesh thickness. Whereas cross $\mathrm{L}_{3} \times \mathrm{T}_{2}$, fruit yield was found to be significantly and positively correlated with node to first male flower, node to first female flower, fruit weight, fruit length and fruit diameter. Path coefficient analysis showed that fruit weight in $\mathrm{L}_{3} \mathrm{xT}_{1}$ cross has contributed the maximum positive direct effect whereas cross $\mathrm{L}_{3} \mathrm{xT}_{2}$ fruit diameter contributed the maximum positive direct effect in F5 generation. 
hemorrhoids, leprosy and splantis. The juice of fresh leaves is useful in granular conjunctivitis for children. The seeds possess purgative and emetic properties (Rahman et al., 2008). Every $100 \mathrm{~g}$ of edible portion of ridge gourd contains $0.5 \mathrm{~g}$ of fiber, 0.5 percent of protein, 0.34 percent of carbohydrate, $37 \mathrm{mg}$ of carotene, $5.0 \mathrm{mg}$ of vitamin C, $18 \mathrm{mg}$ of calcium and $0.5 \mathrm{mg}$ of iron. Besides used as vegetable, it is also used in industries for cleaning and scrubbing machines.

It is also compressed and made into soles for chappals is Japan. Ridge gourd being a monoecious and cross pollinated crop and it exhibits considerable heterozygosity in population and does not suffer much due to inbreeding depression resulting is natural variability in the population.

Thus provides ample scope for exploitation of existing variability on commercial scale to increase the production and productivity (Narasannavar et al., 2014). Most of the ridge gourd hybrids released from India has large sized fruits which are not preferred by a consumer and it affects the marketability as vegetable. The role of genetic variability in crop is of paramount importance in selecting the best genotypes for making rapid improvement in yield and related characters as well as to select the most potential parents for making the hybridization programme successful. Therefore, the present investigation on genetic variability, correlation and path analysis in $F_{5}$ generation of ridge gourd for growth, yield and quality were undertaken during the year $2018-2019$.

\section{Materials and Methods}

The present study was carried out at College Orchard, Department of Horticulture, Agricultural College and Research Institute, Tamil Nadu Agricultural University, Madurai, Tamil Nadu during December 2018 to April
2019. The aim of the present investigation is to develop medium size, high yielding and good quality variety suitable for Madurai condition. The crosses viz., $\mathrm{L}_{3} \mathrm{xT}_{1}$ (Virudhunagar Local x Periyakottai Local) and $\mathrm{L}_{3} \mathrm{xT}_{2}$ (Virudhunagar Local $\mathrm{x}$ Alathur Local) were evaluated along with their parents. The present study was laid out is a Randomized Block Design (RBD) with two replications for $\mathrm{F}_{5}$ generation. The spacing adopted for the study is $2 \mathrm{~m} \times 2 \mathrm{~m}$. From each replication 95 pits were taken and two plants per pit were maintained in each crosses and their parents and accounted for a total population of 190 plants in $\mathrm{F}_{5}$ generation.

The observations on vine length $(\mathrm{cm})$, days to first male flowering, days to first female flowering, node to first male flower, node to first female flower, number of fruits per plant, sex ratio, days to first harvest, fruit weight $(\mathrm{kg})$, fruit length $(\mathrm{cm})$, fruit diameter $(\mathrm{cm})$, rind thickness $(\mathrm{mm})$, flesh thickness $(\mathrm{mm})$, fruit yield per plant $(\mathrm{kg})$, estimated fruit yield per hectare (t/ha), total soluble solids (TSS) $\left({ }^{\circ}\right.$ Brix), total crude fibre content $(\mathrm{mg})$ were recorded.

The data's recorded were statistically analysed of variance of phenotypic coefficient of variation (PCV), genotypic coefficient of variation $(\mathrm{GCV})$, heritability, genetic advance as percentage of mean, correlation studies (AlJibouri et al., (1958) and path co- efficient analysis (Dewey and Lu, 1959) to partition the genotypic correlation coefficient into measures direct and indirect effects.

\section{Results and Discussion}

The extent of variability present in the selected genotype of ridge gourd in $F_{5}$ generation from two crosses viz., $\mathrm{L}_{3} \mathrm{XT}_{1}$ and $\mathrm{L}_{3} \mathrm{xT}_{2}$ were measured for variability, heritability, genetic advance as percentage of mean are presented in Table 1 and 2. 


\section{Genotypic and phenotypic variability}

The analysis of variance revealed significant differences among the two crosses of ridge gourd in F5 generation. The results revealed selection for vine length in the crosses $\mathrm{L}_{3} \mathrm{XT}_{1}$ (4.39; 3.63) and $\mathrm{L}_{3} \times \mathrm{T}_{2}(5.25$; 4.65), days to first male flowering $\mathrm{L}_{3} \mathrm{XT}_{1}(8.04 ; 7.18)$ and $\mathrm{L}_{3} \mathrm{xT}_{2} \quad(7.23 ; 6.51)$, days to first female flowering $\mathrm{L}_{3} \mathrm{xT}_{1}(6.04 ; 5.32)$ and $\mathrm{L}_{3} \mathrm{xT}_{2}(5.95$; 5.02), node to first male flower $\mathrm{L}_{3} \times \mathrm{T}_{1}(8.45$; 5.12) and $\mathrm{L}_{3} \mathrm{xT}_{2}(8.50 ; 6.99)$, sex ratio $\mathrm{L}_{3} \mathrm{xT}_{1}$ (5.03; 4.09) and $\mathrm{L}_{3} \times \mathrm{T}_{2}(4.84 ; 4.57)$, days to first harvest $\mathrm{L}_{3} \times \mathrm{T}_{1}(7.39 ; 5.96)$ and $\mathrm{L}_{3} \times \mathrm{T}_{2}$ (7.82; 6.49), fruit diameter in crosses $\mathrm{L}_{3} \times \mathrm{T}_{1}$ (9.21; 5.48) and $\mathrm{L}_{3} \times \mathrm{T}_{2}(6.05 ; 4.18)$, rind thickness $\mathrm{L}_{3} \mathrm{xT}_{1}(24.29 ; 22.01)$ and $\mathrm{L}_{3} \mathrm{xT}_{2}$ (9.13; 6.98), total soluble solids $\mathrm{L}_{3} \mathrm{xT}_{1}$ (4.31; 3.95) and $\mathrm{L}_{3} \mathrm{xT}_{2}(9.82 ; 7.80)$, total crude fibre content $\mathrm{L}_{3} \mathrm{xT}_{1}(9.34 ; 8.28)$ and $\mathrm{L}_{3} \mathrm{xT}_{2}(7.44$; 5.53) would not be effective due to low PCV and GCV. This agrees with the finding Karthik et al., (2017), Samadia (2011) in ridge gourd and Dey et al., (2009), Puddan (2000) in bitter gourd. This indicates selection resulted in attaining homozygosity and further selection will not alter this trait.

The traits node to first female flower in the crosses $\mathrm{L}_{3} \mathrm{XT}_{1}(17.70 ; 16.13)$ and $\mathrm{L}_{3} \mathrm{XT}_{2}$ $(17.02 ; 15.86)$, number of fruits per plant $\mathrm{L}_{3} \mathrm{XT}_{1} \quad(15.24 ; 14.47)$ and $\mathrm{L}_{3} \times \mathrm{T}_{2}$ (16.59; 15.90), fruit weight $\mathrm{L}_{3} \times \mathrm{T}_{1}(13.78 ; 12.25)$ and $\mathrm{L}_{3} \mathrm{XT}_{2} \quad$ (14.09; 10.11), fruit length $\mathrm{L}_{3} \mathrm{xT}_{1}$ $(19.07 ; 16.60)$ and $\mathrm{L}_{3} \times \mathrm{T}_{2}(11.05 ; 10.51)$, flesh thickness $\mathrm{L}_{3} \times \mathrm{T}_{1}(14.39 ; 12.85)$ and $\mathrm{L}_{3} \mathrm{xT}_{2}$ (13.34; 11.56), fruit yield per plant $\mathrm{L}_{3} \mathrm{XT}_{1} \quad(15.24 ; 12.42)$ and $\mathrm{L}_{3} \mathrm{xT}_{2}$ (18.79; 17.21), estimated fruit yield per hectare $\mathrm{L}_{3} \mathrm{XT}_{1}$ $(16.21 ; 13.98)$ and $\mathrm{L}_{3} \mathrm{xT}_{2}(19.09 ; 17.31$ were exhibited moderate PCV and GCV. This was agrees with the findings of Koppad et al., (2015), Samadia (2011) in ridge gourd, Devi and Mariappan (2013) in snake gourd and Dey et al., (2009) in bitter gourd. This indicates the presence of medium amount of variability and improvements of these traits is possible up to an extent in further generation and attain homozygosity.

In case of rind thickness, $\mathrm{L}_{3} \mathrm{xT}_{1}(24.29 ; 22.01)$ cross showed high magnitude of PCV and Koppad et al., (2015) found that high variability was occurred among the genotypes of ridge gourd. This confirms the presence of diverse genotypes in $F_{5}$ in these cross and improvement through selection is possible in further selection.

\section{Heritability and genetic advance}

Heritability and genetic advance as percentage of mean are presented in Table 1 and 2. In the present study high heritability coupled with high genetic advance were recorded for the traits node to first male flower, node to first female flower, number of fruits per plant, fruit weight, fruit length, rind thickness, flesh thickness, fruit yield per plant and estimated fruit yield per hectare in both the crosses. This confirm the presence of additive gene action and the traits are less influenced by environment and selecting the genotypes based on such characters could be worthwhile which agrees with Samadia (2011) in ridge gourd, Sahitya (2001) in snake gourd and Dey et al., (2009) in bitter gourd.

High heritability coupled with moderate genetic advance was recorded for the traits days to first male flowering, days to first harvest and total crude fibre content in both the crosses. This might be due to homozygous line could be developed through continuous selection process and these results are similar to the findings of Choudhary and Kumar (2011) in ridge gourd and Kanimozhi et al., (2015) in wax gourd.

High heritability coupled with low genetic advance was recorded for the traits vine length, days to female flowering and sex ratio 
in both the crosses. This indicated the presence of certain degree of non-additive gene effect and was supported by findings in ridge gourd (Choudhary and Kumar, 2011, Samadia, 2011) and (Kanimozhi et al., 2015) in wax gourd.

\section{Correlation coefficient analysis}

It was necessary to determine the magnitude and direction of relationship between yield and its components for the improvement of yield in ridge gourd. The sixteen traits in $\mathrm{F}_{5}$ generation of $\mathrm{L}_{3} \mathrm{xT}_{1}$ and $\mathrm{L}_{3} \mathrm{xT}_{2}$ were considered for correlation analysis. Crosses $\mathrm{L}_{3} \mathrm{xT}_{1}$ and $\mathrm{L}_{3} \mathrm{xT}_{2}$ had registered correlation coefficients between yield and its components estimated were given in Table $3 \& 4$. The cross $\mathrm{L}_{3} \mathrm{xT}_{1}$, fruit yield was found to be significantly and positively correlated with node to first male flower $(0.910)$, node to first female flower (0.552), days to first harvest (0.458), fruit weight (0.772), flesh thickness $(0.660)$ and number of fruits per plant (0.455).

Table.1 Estimates of mean, components of variance, heritability and genetic advance for growth, flowering, yield and quality parameters in Virudhunagar local x Periyakottai local $\left(\mathrm{L}_{3} \mathrm{XT}_{1}\right)$

\begin{tabular}{|c|c|c|c|c|c|c|}
\hline S. No & Characters & Mean & $\begin{array}{l}\text { PCV } \\
(\%)\end{array}$ & $\begin{array}{l}\text { GCV } \\
(\%)\end{array}$ & $\mathbf{h}^{2}(\%)$ & GAM \\
\hline A. & Growth traits & & & & & \\
\hline 1. & Vine length(m) & 7.76 & 4.39 & 3.63 & 84.73 & 7.42 \\
\hline B. & \multicolumn{6}{|c|}{ Flowering Traits } \\
\hline 1. & Days to $1^{\text {st }}$ male flowering & 26.43 & 8.04 & 7.18 & 79.43 & 13.16 \\
\hline 2. & Days to $1^{\text {st }}$ female flowering & 34.17 & 6.04 & 5.32 & 73.34 & 8.72 \\
\hline 3. & Node to $1^{\text {st }}$ male flower & 5.87 & 8.45 & 5.12 & 78.48 & 26.80 \\
\hline 4. & Node to $1^{\text {st }}$ female flower & 16.28 & 17.70 & 16.13 & 84.56 & 31.68 \\
\hline 5. & Sex ratio & 5.79 & 5.03 & 4.09 & 79.73 & 7.29 \\
\hline 6. & Days to first harvest & 65.17 & 7.39 & 5.96 & 86.63 & 13.42 \\
\hline C. & \multicolumn{6}{|c|}{ Yield Traits } \\
\hline 1. & Number of fruits per plant & 12.19 & 15.24 & 14.47 & 93.47 & 32.28 \\
\hline 2. & Fruit weight $(\mathrm{kg})$ & 0.265 & 13.78 & 12.25 & 78.65 & 25.03 \\
\hline 3. & Fruit length $(\mathrm{cm})$ & 27.50 & 19.07 & 16.60 & 89.59 & 34.92 \\
\hline 4. & Fruit diameter $(\mathrm{cm})$ & 4.38 & 9.21 & 5.48 & 54.45 & 19.21 \\
\hline 5. & Rind thickness (cm) & 0.41 & 24.29 & 22.01 & 94.59 & 47.52 \\
\hline 6. & Flesh thickness (cm) & 3.36 & 14.39 & 12.85 & 86.12 & 27.53 \\
\hline 7. & Fruit yield per plant (kg) & 4.42 & 15.24 & 12.42 & 84.84 & 22.58 \\
\hline 8. & $\begin{array}{l}\text { Estimated fruit yield per hectare } \\
\qquad(\mathrm{t} / \mathrm{ha})\end{array}$ & 19.76 & 16.21 & 13.98 & 89.14 & 35.18 \\
\hline D. & \multicolumn{6}{|c|}{ Quality Traits } \\
\hline 1. & Total Soluble Solids (TSS) & 3.89 & 4.31 & 3.95 & 94.65 & 24.63 \\
\hline 2. & $\begin{array}{l}\text { Total Crude Fibre Content } \\
\text { (mg) }\end{array}$ & 0.48 & 9.34 & 8.28 & 78.62 & 15.40 \\
\hline
\end{tabular}

$\mathrm{PCV}=$ Phenotypic coefficient of variance

$\mathrm{GCV}=$ Genotypic coefficient of variance

$\mathrm{h}^{2}=$ Heritability (broad sense) $\quad$ GAM $=$ Genetic advance (per cent mean) 
Table.2 Estimates of mean, components of variance, heritability and genetic advance for growth, flowering, yield and quality parameters in Virudhunagar local x Alathur local $\left(\mathrm{L}_{3} \mathrm{XT}_{2}\right)$

\begin{tabular}{|c|c|c|c|c|c|c|}
\hline S. No. & Characters & Mean & $\begin{array}{l}\text { PCV } \\
(\%)\end{array}$ & $\begin{array}{l}\text { GCV } \\
(\%)\end{array}$ & $h^{2}(\%)$ & GAM \\
\hline A. & \multicolumn{6}{|c|}{ Growth traits } \\
\hline 1. & Vine length(m) & 7.12 & 5.25 & 4.65 & 77.21 & 8.49 \\
\hline B. & \multicolumn{6}{|c|}{ Flowering Traits } \\
\hline 1. & Days to $1^{\text {st }}$ male flowering & 29.42 & 7.23 & 6.51 & 75.21 & 12.40 \\
\hline 2. & Days to $1^{\text {st }}$ female flowering & 37.28 & 5.95 & 5.02 & 61.12 & 11.82 \\
\hline 3. & Node to $1^{\text {st }}$ male flower & 5.80 & 8.50 & 6.99 & 75.69 & 28.04 \\
\hline 4. & Node to $1^{\text {st }}$ female flower & 17.14 & 17.02 & 15.86 & 78.4 & 26.48 \\
\hline 5. & Sex ratio & 5.16 & 4.84 & 4.57 & 72.17 & 5.45 \\
\hline 6. & Days to first harvest & 68.35 & 7.82 & 6.49 & 69.56 & 16.21 \\
\hline C. & \multicolumn{6}{|c|}{ Yield Traits } \\
\hline 1. & Number of fruits per plant & 10.54 & 16.59 & 15.90 & 91.24 & 24.39 \\
\hline 2. & Fruit weight (kg) & 0.278 & 14.09 & 10.11 & 68.87 & 28.26 \\
\hline 3. & Fruit length $(\mathrm{cm})$ & 28.52 & 11.05 & 10.51 & 95.91 & 34.40 \\
\hline 4. & Fruit diameter $(\mathrm{cm})$ & 4.46 & 6.05 & 4.18 & 51.93 & 24.02 \\
\hline 5. & Rind thickness (cm) & 0.46 & 9.13 & 6.98 & 77.89 & 34.03 \\
\hline 6. & Flesh thickness $(\mathrm{cm})$ & 3.54 & 13.34 & 11.56 & 89.17 & 26.13 \\
\hline 7. & Fruit yield per plant (kg) & 3.95 & 18.79 & 17.21 & 87.55 & 19.53 \\
\hline 8. & $\begin{array}{l}\text { Estimated fruit yield per hectare } \\
\qquad(\mathrm{t} / \mathrm{ha})\end{array}$ & 16.57 & 19.09 & 17.31 & 85.29 & 33.04 \\
\hline D. & \multicolumn{6}{|c|}{ Quality Traits } \\
\hline 1. & Total Soluble Solids (TSS) & 3.32 & 9.82 & 7.80 & 84.28 & 13.29 \\
\hline 2. & Total crude fibre content (mg) & 0.46 & 7.44 & 5.53 & 79.28 & 13.92 \\
\hline & $\begin{array}{l}=\text { Phenotypic coefficient of variance } \\
=\text { Heritability (broad sense) }\end{array}$ & & $\begin{array}{l}\mathrm{CV}=\mathrm{G} \\
\mathrm{AM}=\mathrm{C}\end{array}$ & $\begin{array}{l}\text { pic cc } \\
\mathrm{c} \mathrm{adv}\end{array}$ & ent of $v$ & \\
\hline
\end{tabular}


Table.3 Correlation coefficients of the cross Virudhunagar local x Periyakottai local $\left(\mathrm{L}_{3} \mathrm{x} \mathrm{T}_{1}\right)$ in $\mathrm{F}_{5}$ generation

\begin{tabular}{|c|c|c|c|c|c|c|c|c|c|c|c|c|c|c|c|c|}
\hline & $\begin{array}{c}\text { Vine } \\
\text { length }\end{array}$ & $\begin{array}{l}\text { Days to } \\
\text { first male } \\
\text { flowering }\end{array}$ & $\begin{array}{c}\text { Days to } \\
\text { first } \\
\text { female } \\
\text { flowering }\end{array}$ & $\begin{array}{l}\text { Node to } \\
\text { first male } \\
\text { flowering }\end{array}$ & $\begin{array}{c}\text { Node to } \\
\text { first } \\
\text { female } \\
\text { flowering }\end{array}$ & $\begin{array}{c}\text { Days to } \\
\text { first } \\
\text { harvest }\end{array}$ & $\begin{array}{c}\text { Fruit } \\
\text { weight }\end{array}$ & $\begin{array}{l}\text { Fruit } \\
\text { length }\end{array}$ & $\begin{array}{c}\text { Fruit } \\
\text { diameter }\end{array}$ & $\begin{array}{c}\text { Rind } \\
\text { thickness }\end{array}$ & $\begin{array}{c}\text { Flesh } \\
\text { thickness }\end{array}$ & $\begin{array}{c}\text { Number } \\
\text { of fruits } \\
\text { per } \\
\text { plant }\end{array}$ & $\begin{array}{c}\text { Sex } \\
\text { ratio }\end{array}$ & $\begin{array}{c}\text { Total } \\
\text { soluble } \\
\text { solids }\end{array}$ & $\begin{array}{l}\text { Total } \\
\text { crude } \\
\text { fibre } \\
\text { content }\end{array}$ & $\begin{array}{l}\text { Fruit } \\
\text { yield }\end{array}$ \\
\hline Vine length & 1.000 & -0.893 & -0.252 & $0.825 * *$ & $0.559 *$ & -0.762 & 0.350 & -0.412 & -0.927 & 0.108 & -0.964 & 0.465 & -0.683 & -0.728 & -0.104 & -0.335 \\
\hline $\begin{array}{l}\text { Days to first } \\
\text { male flowering }\end{array}$ & & 1.000 & $0.842 * *$ & $0.723 * *$ & 0.247 & 0.208 & 0.365 & 0.414 & $0.567 * *$ & 0.390 & $0.784 * *$ & 0.402 & 0.330 & 0.275 & -0.069 & 0.455 \\
\hline $\begin{array}{l}\text { Days to first } \\
\text { female } \\
\text { flowering }\end{array}$ & & & 1.000 & $0.947 * *$ & $0.447 *$ & 0.379 & 0.213 & 0.440 & 0.306 & 0.440 & 0.192 & $0.701 * *$ & 0.397 & 0.253 & -0.087 & 0.120 \\
\hline $\begin{array}{l}\text { Node to first } \\
\text { male flowering }\end{array}$ & & & & 1.000 & $0.511^{*}$ & -0.039 & 0.367 & -0.745 & -0.439 & -0.226 & -0.422 & 0.237 & 0.142 & 0.204 & -0.054 & $0.910 * *$ \\
\hline $\begin{array}{l}\text { Node to first } \\
\text { female } \\
\text { flowering }\end{array}$ & & & & & 1.000 & $0.469 *$ & -0.287 & 0.287 & 0.023 & -0.420 & 0.262 & -1.015 & 0.205 & -0.321 & -0.097 & $0.552 * *$ \\
\hline $\begin{array}{c}\text { Days to first } \\
\text { harvest }\end{array}$ & & & & & & 1.000 & $0.461 *$ & 0.337 & 0.055 & -0.622 & 0.143 & $0.782 * *$ & $0.731 * *$ & -1.103 & 0.069 & $0.458^{*}$ \\
\hline Fruit weight & & & & & & & 1.000 & $0.802 * *$ & 0.255 & $0.510^{*}$ & $0.875 * *$ & -1.219 & 0.342 & -0.985 & 0.017 & $0.772 * *$ \\
\hline Fruit length & & & & & & & & 1.000 & $0.739 * *$ & 0.259 & 0.286 & -0.085 & $0.971 * *$ & -0.896 & 0.078 & 0.400 \\
\hline Fruit diameter & & & & & & & & & 1.000 & 0.270 & $0.987 * *$ & 0.200 & 0.024 & 0.105 & 0.032 & 0.164 \\
\hline Rind thickness & & & & & & & & & & 1.000 & 0.421 & $0.770 * *$ & -0.443 & -0.130 & 0.029 & 0.427 \\
\hline Flesh thickness & & & & & & & & & & & 1.000 & $0.606 * *$ & 0.069 & 0.374 & 0.106 & $0.660 * *$ \\
\hline $\begin{array}{c}\text { Number of } \\
\text { fruits per plant }\end{array}$ & & & & & & & & & & & & 1.000 & $0.593 * *$ & -0.467 & 0.086 & $0.455^{* * *}$ \\
\hline Sex ratio & & & & & & & & & & & & & 1.000 & -0.721 & -0.111 & 0.188 \\
\hline $\begin{array}{l}\text { Total soluble } \\
\text { solids }\end{array}$ & & & & & & & & & & & & & & 1.000 & 0.022 & -0.528 \\
\hline $\begin{array}{l}\text { Total crude } \\
\text { fibre content }\end{array}$ & & & & & & & & & & & & & & & 1.000 & 0.074 \\
\hline Fruit yield & & & & & & & & & & & & & & & & 1.000 \\
\hline
\end{tabular}


Table.4 Correlation coefficients of the cross Virudhunagar local x Alathur local $\left(\mathrm{L}_{3} \mathrm{xT}_{2}\right)$ in $\mathrm{F}_{5}$ generation

\begin{tabular}{|c|c|c|c|c|c|c|c|c|c|c|c|c|c|c|c|c|}
\hline & $\begin{array}{c}\text { Vine } \\
\text { length }\end{array}$ & $\begin{array}{l}\text { Days to } \\
\text { first male } \\
\text { flowering }\end{array}$ & $\begin{array}{c}\text { Days to } \\
\text { first } \\
\text { female } \\
\text { flowering }\end{array}$ & $\begin{array}{l}\text { Node to } \\
\text { first male } \\
\text { flowering }\end{array}$ & $\begin{array}{c}\text { Node to } \\
\text { first } \\
\text { female } \\
\text { flowering }\end{array}$ & $\begin{array}{c}\text { Days to } \\
\text { first } \\
\text { harvest }\end{array}$ & $\begin{array}{c}\text { Fruit } \\
\text { weight }\end{array}$ & $\begin{array}{l}\text { Fruit } \\
\text { length }\end{array}$ & $\begin{array}{c}\text { Fruit } \\
\text { diameter }\end{array}$ & $\begin{array}{c}\text { Rind } \\
\text { thickness }\end{array}$ & $\begin{array}{c}\text { Flesh } \\
\text { thickness }\end{array}$ & $\begin{array}{c}\text { Number } \\
\text { of fruits } \\
\text { per } \\
\text { plant }\end{array}$ & Sex ratio & $\begin{array}{c}\text { Total } \\
\text { soluble } \\
\text { solids }\end{array}$ & $\begin{array}{c}\text { Total } \\
\text { crude } \\
\text { fibre } \\
\text { content }\end{array}$ & $\begin{array}{l}\text { Fruit } \\
\text { yield }\end{array}$ \\
\hline Vine length & 1.000 & -0.024 & 0.157 & -0.391 & -0.624 & 0.124 & 0.115 & 0.010 & 0.217 & -0.193 & 0.200 & 0.120 & -0.179 & 0.058 & -0.135 & 0.395 \\
\hline $\begin{array}{l}\text { Days to first } \\
\text { male flowering }\end{array}$ & & 1.000 & 0.270 & -0.183 & 0.110 & 0.056 & 0.102 & 0.124 & -0.154 & -0.081 & -0.114 & 0.196 & 0.083 & -0.198 & -0.010 & 0.156 \\
\hline $\begin{array}{l}\text { Days to first } \\
\text { female } \\
\text { flowering }\end{array}$ & & & 1.000 & 0.015 & 0.127 & -0.136 & 0.261 & 0.165 & 0.280 & -0.050 & 0.019 & 0.110 & $0.683^{*}$ & -0.176 & 0.028 & -0.141 \\
\hline $\begin{array}{c}\text { Node to first } \\
\text { male flowering }\end{array}$ & & & & 1.000 & $0.640^{*}$ & $0.736^{* *}$ & 0.218 & -0.230 & -0.258 & 0.224 & 0.054 & 0.091 & -0.019 & 0.158 & 0.170 & $0.721 * *$ \\
\hline $\begin{array}{l}\text { Node to first } \\
\text { female } \\
\text { flowering }\end{array}$ & & & & & 1.000 & 0.176 & 0.139 & -0.084 & 0.177 & 0.106 & 0.117 & 0.124 & -0.128 & 0.025 & -0.082 & $0.778 * *$ \\
\hline $\begin{array}{c}\text { Days to first } \\
\text { harvest }\end{array}$ & & & & & & 1.000 & $0.878 * *$ & -0.100 & $0.852 * *$ & -0.058 & -0.233 & -0.188 & 0.056 & 0.016 & -0.036 & 0.121 \\
\hline Fruit weight & & & & & & & 1.000 & $0.750 * *$ & $0.756 * *$ & -0.046 & $0.687 *$ & 0.360 & $0.624 *$ & 0.146 & 0.146 & $0.598 *$ \\
\hline Fruit length & & & & & & & & 1.000 & 0.149 & -0.067 & -0.172 & 0.543 & 0.131 & -0.220 & -0.043 & $0.623 *$ \\
\hline Fruit diameter & & & & & & & & & 1.000 & 0.167 & $0.716^{* *}$ & 0.387 & 0.240 & -0.106 & -0.022 & $0.760 * *$ \\
\hline Rind thickness & & & & & & & & & & 1.000 & 0.102 & $0.724 * *$ & -0.052 & -0.028 & 0.112 & 0.246 \\
\hline Flesh thickness & & & & & & & & & & & 1.000 & 0.186 & 0.294 & 0.080 & 0.019 & 0.470 \\
\hline $\begin{array}{l}\text { Number of } \\
\text { fruits per plant }\end{array}$ & & & & & & & & & & & & 1.000 & -0.572 & -0.214 & 0.047 & 0.512 \\
\hline Sex ratio & & & & & & & & & & & & & 1.000 & 0.025 & -0.082 & 0.153 \\
\hline $\begin{array}{l}\text { Total soluble } \\
\text { solids }\end{array}$ & & & & & & & & & & & & & & 1.000 & 0.095 & -0.121 \\
\hline $\begin{array}{c}\text { Total crude } \\
\text { fibre content }\end{array}$ & & & & & & & & & & & & & & & 1.000 & 0.162 \\
\hline Fruit yield & & & & & & & & & & & & & & & & 1.000 \\
\hline
\end{tabular}


Table.5 Path coefficient analysis of cross Virudhunagar local x Periyakottai local $\left(\mathrm{L}_{3} \mathrm{xT}_{1}\right)$ in $\mathrm{F}_{5}$ generation

\begin{tabular}{|c|c|c|c|c|c|c|c|c|c|c|c|c|c|c|c|c|}
\hline & $\begin{array}{c}\text { Vine } \\
\text { length }\end{array}$ & $\begin{array}{c}\text { Days to first } \\
\text { male } \\
\text { flowering }\end{array}$ & $\begin{array}{l}\text { Days to firs } \\
\text { female } \\
\text { flowering }\end{array}$ & $\begin{array}{l}\text { Node to firs } \\
\text { male } \\
\text { flowering }\end{array}$ & $\begin{array}{l}\text { Node to first } \\
\text { female } \\
\text { flowering }\end{array}$ & $\begin{array}{c}\text { Days to } \\
\text { first } \\
\text { harvest }\end{array}$ & $\begin{array}{c}\text { Fruit } \\
\text { weight }\end{array}$ & $\begin{array}{c}\text { Fruit } \\
\text { length }\end{array}$ & $\begin{array}{c}\text { Fruit } \\
\text { diameter }\end{array}$ & $\begin{array}{c}\text { Rind } \\
\text { thickness }\end{array}$ & $\begin{array}{c}\text { Flesh } \\
\text { thickness }\end{array}$ & $\begin{array}{c}\text { Number of } \\
\text { fruits per } \\
\text { plant }\end{array}$ & Sex ratio & $\begin{array}{c}\text { Total } \\
\text { soluble } \\
\text { solids }\end{array}$ & $\begin{array}{c}\text { Total } \\
\text { crude } \\
\text { fibre } \\
\text { content }\end{array}$ & Fruit yield \\
\hline Vine length & 0.938 & 0.470 & -0.650 & -0.207 & -0.557 & 0.050 & 0.012 & -0.176 & 0.739 & 0.344 & -0.043 & 0.037 & -0.035 & 0.007 & -0.012 & -0.335 \\
\hline $\begin{array}{c}\text { Days to first } \\
\text { male } \\
\text { flowering }\end{array}$ & -0.773 & 0.307 & 0.034 & 0.943 & -0.157 & -0.030 & 0.001 & 0.019 & 0.316 & -0.232 & 0.036 & 0.054 & -0.012 & 0.015 & -0.003 & 0.455 \\
\hline $\begin{array}{l}\text { Days to first } \\
\text { female } \\
\text { flowering }\end{array}$ & 0.003 & -0.003 & 0.041 & 0.027 & -0.038 & 0.058 & -0.052 & 0.014 & 0.011 & -0.013 & -0.014 & 0.129 & 0.034 & -0.120 & -0.007 & 0.120 \\
\hline $\begin{array}{c}\text { Node to first } \\
\text { male } \\
\text { flowering }\end{array}$ & -0.036 & -0.019 & 0.027 & 0.845 & -0.066 & -0.017 & -0.256 & -0.011 & -0.113 & -0.017 & 0.335 & -0.041 & -0.058 & 0.056 & -0.003 & -0.910 \\
\hline $\begin{array}{l}\text { Node to first } \\
\text { female } \\
\text { flowering }\end{array}$ & 0.031 & 0.020 & -0.049 & -0.077 & -0.073 & 0.067 & -0.029 & -0.016 & -0.106 & 0.040 & -0.072 & -0.153 & -0.076 & 0.033 & 0.006 & 0.552 \\
\hline $\begin{array}{c}\text { Days to first } \\
\text { harvest }\end{array}$ & 0.004 & -0.030 & 0.098 & 0.036 & 0.120 & -0.024 & 0.065 & 0.034 & -0.020 & -0.021 & -0.016 & 0.034 & 0.186 & 0.065 & 0.015 & 0.458 \\
\hline Fruit weight & 0.021 & -0.054 & 0.029 & -0.015 & 0.018 & 0.021 & 1.131 & 0.091 & 0.022 & -0.011 & 0.037 & 0.073 & 0.019 & 0.079 & 0.007 & 0.772 \\
\hline Fruit length & 0.014 & -0.062 & 0.015 & -0.155 & 0.195 & 0.626 & 0.536 & 1.126 & 0.102 & -0.058 & 0.045 & 0.096 & 0.059 & -0.213 & -0.010 & 0.400 \\
\hline $\begin{array}{c}\text { Fruit } \\
\text { diameter }\end{array}$ & 0.004 & 0.011 & -0.009 & 0.012 & 0.046 & -0.010 & 0.303 & 0.051 & 1.014 & -0.021 & 0.058 & 0.049 & 0.026 & 0.090 & 0.015 & 0.164 \\
\hline $\begin{array}{c}\text { Rind } \\
\text { thickness }\end{array}$ & -0.021 & -0.011 & 0.021 & 0.052 & 0.038 & -0.026 & 0.021 & 0.032 & -0.013 & -0.124 & 0.036 & 0.025 & 0.045 & 0.075 & 0.025 & 0.427 \\
\hline $\begin{array}{c}\text { Flesh } \\
\text { thickness }\end{array}$ & -0.052 & -0.032 & 0.048 & -0.017 & -0.012 & -0.042 & 0.396 & 0.060 & -0.010 & -0.013 & 0.093 & 0.099 & -0.014 & -0.142 & 0.020 & 0.660 \\
\hline $\begin{array}{c}\text { Number of } \\
\text { fruits per } \\
\text { plant }\end{array}$ & -0.033 & 0.035 & 0.012 & -0.042 & 0.082 & -0.019 & 0.118 & 0.026 & -0.016 & -0.072 & 0.091 & 1.003 & -0.038 & 0.055 & -0.007 & 0.455 \\
\hline Sex ratio & 0.009 & -0.013 & -0.018 & 0.022 & 0.032 & -0.003 & 0.038 & 0.014 & -0.009 & -0.003 & -0.008 & -0.103 & 0.167 & 0.031 & 0.010 & 0.188 \\
\hline $\begin{array}{l}\text { Total soluble } \\
\text { solids }\end{array}$ & 0.022 & -0.056 & 0.041 & -0.072 & 0.056 & 0.021 & 0.065 & 0.090 & 0.057 & 0.057 & 0.034 & -0.010 & -0.020 & -0.663 & 0.020 & -0.528 \\
\hline $\begin{array}{c}\text { Total crude } \\
\text { fibre content }\end{array}$ & 0.006 & -0.005 & 0.005 & 0.001 & -0.005 & -0.006 & 0.008 & 0.072 & -0.116 & -0.014 & -0.017 & 0.009 & -0.017 & 0.016 & -0.102 & 0.074 \\
\hline
\end{tabular}

$$
\text { RESIDUAL EFFECT }=\mathbf{0 . 0 5 0}
$$


Table.6 Path coefficient analysis of cross Virudhunagar local x Alathur local $\left(\mathrm{L}_{3} \mathrm{x} \mathrm{T}_{2}\right)$ in $\mathrm{F}_{5}$ generation

\begin{tabular}{|c|c|c|c|c|c|c|c|c|c|c|c|c|c|c|c|c|}
\hline & $\begin{array}{c}\text { Vine } \\
\text { length }\end{array}$ & $\begin{array}{c}\text { Days to first } \\
\text { male } \\
\text { flowering }\end{array}$ & $\begin{array}{l}\text { Days to first } \\
\text { female } \\
\text { flowering }\end{array}$ & $\begin{array}{l}\text { Node to first } \\
\text { male } \\
\text { flowering }\end{array}$ & $\begin{array}{l}\text { Node to first } \\
\text { female } \\
\text { flowering }\end{array}$ & $\begin{array}{c}\text { Days to } \\
\text { first } \\
\text { harvest }\end{array}$ & $\begin{array}{c}\text { Fruit } \\
\text { weight }\end{array}$ & $\begin{array}{l}\text { Fruit } \\
\text { length }\end{array}$ & $\begin{array}{c}\text { Fruit } \\
\text { diameter }\end{array}$ & $\begin{array}{c}\text { Rind } \\
\text { thickness }\end{array}$ & $\begin{array}{c}\text { Flesh } \\
\text { thickness }\end{array}$ & $\begin{array}{c}\text { Number of } \\
\text { fruits per } \\
\text { plant }\end{array}$ & Sex ratio & $\begin{array}{c}\text { Total } \\
\text { soluble } \\
\text { solids }\end{array}$ & $\begin{array}{c}\text { Total } \\
\text { crude } \\
\text { fibre } \\
\text { content }\end{array}$ & Fruit yield \\
\hline Vine length & 0.005 & -0.002 & 0.011 & 0.007 & 0.003 & 0.002 & 0.261 & -0.004 & -0.021 & -0.018 & 0.036 & 0.213 & -0.002 & -0.015 & -0.003 & 0.395 \\
\hline $\begin{array}{c}\text { Days to first } \\
\text { male } \\
\text { flowering }\end{array}$ & 0.006 & -0.015 & 0.086 & -0.005 & -0.007 & 0.012 & 0.543 & 0.027 & 0.054 & 0.038 & -0.021 & -0.027 & -0.004 & -0.027 & 0.002 & 0.156 \\
\hline $\begin{array}{c}\text { Days to first } \\
\text { female } \\
\text { flowering }\end{array}$ & 0.009 & -0.024 & -0.088 & -0.008 & 0.017 & -0.026 & 0.227 & -0.024 & 0.014 & 0.032 & -0.029 & -0.642 & -0.008 & 0.009 & 0.001 & -0.141 \\
\hline $\begin{array}{c}\text { Node to first } \\
\text { male } \\
\text { flowering }\end{array}$ & -0.039 & 0.038 & 0.028 & -0.015 & -0.021 & 0.068 & 1.216 & 0.116 & 0.217 & 0.040 & -0.096 & -0.299 & 0.012 & -0.019 & 0.001 & 0.721 \\
\hline $\begin{array}{l}\text { Node to first } \\
\text { female } \\
\text { flowering }\end{array}$ & 0.011 & -0.013 & -0.029 & -0.005 & 0.213 & -0.047 & 0.286 & 0.043 & 0.038 & -0.019 & -0.058 & 0.625 & 0.019 & 0.027 & 0.002 & 0.778 \\
\hline $\begin{array}{c}\text { Days to first } \\
\text { harvest }\end{array}$ & -0.002 & -0.025 & 0.024 & -0.019 & 0.021 & 0.675 & 0.606 & 0.098 & -0.105 & -0.067 & 0.013 & 0.082 & -0.016 & -0.015 & 0.005 & 0.121 \\
\hline Fruit weight & -0.095 & 0.031 & 0.065 & -0.022 & 0.014 & 0.046 & 1.212 & -0.089 & -0.372 & -0.049 & 0.183 & 0.219 & -0.029 & -0.045 & 0.002 & 0.598 \\
\hline Fruit length & 0.059 & -0.012 & 0.044 & 0.018 & 0.021 & 0.034 & 0.652 & 1.321 & 0.589 & 0.051 & 0.088 & 0.304 & -0.021 & 0.036 & -0.003 & 0.623 \\
\hline $\begin{array}{c}\text { Fruit } \\
\text { diameter }\end{array}$ & 0.007 & 0.019 & -0.021 & 0.011 & -0.015 & 0.063 & 0.431 & 0.051 & 1.384 & -0.027 & 0.143 & 0.131 & -0.008 & -0.021 & 0.003 & 0.760 \\
\hline $\begin{array}{c}\text { Rind } \\
\text { thickness }\end{array}$ & -0.005 & -0.016 & -0.013 & -0.010 & 0.006 & -0.027 & -0.324 & 0.128 & 0.313 & 0.122 & -0.290 & 0.146 & 0.005 & 0.057 & 0.004 & 0.246 \\
\hline $\begin{array}{c}\text { Flesh } \\
\text { thickness }\end{array}$ & 0.011 & 0.024 & -0.082 & 0.003 & 0.024 & 0.065 & 1.146 & 0.222 & 0.251 & -0.116 & 0.319 & -0.542 & -0.014 & -0.045 & 0.002 & 0.470 \\
\hline $\begin{array}{c}\text { Number of } \\
\text { fruits per } \\
\text { plant }\end{array}$ & 0.025 & -0.013 & 0.054 & -0.014 & 0.019 & -0.172 & 0.352 & 0.073 & -0.028 & 0.039 & 0.037 & 1.070 & 0.021 & 0.017 & -0.002 & 0.512 \\
\hline Sex ratio & -0.001 & 0.001 & 0.063 & 0.005 & 0.011 & 0.009 & -0.127 & -0.015 & -0.421 & 0.032 & 0.056 & 0.132 & -0.097 & -0.029 & -0.003 & 0.153 \\
\hline $\begin{array}{l}\text { Total soluble } \\
\text { solids }\end{array}$ & 0.016 & -0.021 & 0.014 & -0.013 & 0.042 & 0.051 & 0.090 & 0.082 & -0.011 & -0.014 & 0.154 & -0.052 & -0.012 & -0.048 & 0.004 & -0.121 \\
\hline $\begin{array}{c}\text { Total crude } \\
\text { fibre content }\end{array}$ & -0.006 & 0.005 & -0.005 & -0.002 & -0.002 & 0.009 & 0.008 & -0.071 & 0.003 & 0.017 & -0.008 & -0.017 & 0.008 & 0.016 & -0.010 & 0.028 \\
\hline
\end{tabular}

RESIDUAL EFFECT $=\mathbf{0 . 0 7 1}$ 
Whereas the cross $\mathrm{L}_{3} \mathrm{xT}_{2}$, fruit yield was found to be significantly and positively correlated with node to first male flower $(0.721)$, node to first female flower (0.778), fruit weight (0.598), fruit length $(0.623)$ and fruit diameter (0.760). This indicated that fruit yield can be improved by making selections on the bases of these yield attributing characters. Similar results were reported by Kumar et al., 2007 in bottle gourd; Ananthan and Krishnamoorthy 2017 in ridge gourd, Sampath and Krishnamoorthy 2017 in pumpkin and Rajawat and Collis 2017 in cucumber.

\section{Inter correlations among yield attributing components}

The present study revealed that vine length was exhibited significant and positive correlation with node to first male flower (0.825), node to first female flower (0.559). The trait days to first male flower was exhibited significant and positive correlation with days to first female flower (0.842), node to first male flower (0.723), fruit diameter (0.567) and flesh thickness (0.784) whereas the trait days to first female flower was exhibited significant and positive correlation with node to first male flower (0.947), node to first female flower (0.447) and number of fruits per plant (0.701).

Days to first harvest was found to significant and positive correlation with fruit weight (0.461), number of fruits per plant (0.782) and sex ratio (0.731). Fruit weight was showed significant and positive correlation with fruit length (0.802), rind thickness (0.510) and flesh thickness $(0.875)$.

Fruit length was exhibited significant and positive correlation with fruit diameter (0.739) and sex ratio (0.971) in $\mathrm{L}_{3} \mathrm{XT}_{1}$ cross. These results corroborate the findings of Lakshmi et al., (2000) and Tamilselvi (2010) in pumpkin.
Days to first female flower exhibited significant and positive correlation with sex ratio (0.683). Node to first male flower also showed significant and positive correlation with node to first female flower (0.640) and days to first harvest $(0.736)$. The trait days to first harvest exhibited significant and positive correlation with fruit weight $(0.878)$ and fruit diameter (0.852).

Fruit weight showed significant and positive correlation with fruit length (0.750), fruit diameter (0.756), flesh thickness (0.687) and sex ratio (0.624). Fruit diameter showed significant and positive correlation with flesh thickness (0.716). Rind thickness also exhibited significant and positive correlation with number of fruits per plant (0.724) in $\mathrm{L}_{3} \mathrm{XT}_{2}$ cross and similar results were reported Lakshmi et al., (2000) in pumpkin and Rahman et al., (2002) in snake gourd.

\section{Path coefficient analysis}

Path analysis (Table 5) results revealed that vine length (0.938), days to first male flower (0.307), days to first female flower (0.041), node to first male flower $(0.845)$, fruit weight (1.131), fruit length (1.126), fruit diameter (1.014), flesh thickness (0.093), number of fruits per plant (1.003) and sex ratio (0.167) exhibited significant and positive association with fruit yield $\mathrm{L}_{3} \mathrm{XT}_{1}$, cross. Among sixteen traits, fruit weight showed a highly significant and positive association (1.131) followed by fruit length (1.126), fruit diameter (1.014) and number of fruits per plant (1.003). Regarding path coefficient analysis (Table 6) of the cross $\mathrm{L}_{3} \mathrm{xT}_{2}$ results revealed that vine length $(0.005)$, node to first female flower (0.213), days to first harvest (0.675), fruit weight (1.212), fruit length (1.321), fruit diameter (1.384), rind thickness (0.112), flesh thickness (0.319) and number of fruits per plant (1.070) exhibited significant and positive association with fruit yield. 
Among sixteen traits, fruit diameter (1.384) showed highly significant and positive association followed by fruit length (1.321), fruit weight (1.212) and number of fruits per plant (1.070). From the F5 generation of $\mathrm{L}_{3} \mathrm{XT}_{1}$ and $\mathrm{L}_{3} \mathrm{xT}_{2}$ crosses, fruit length, fruit diameter, fruit weight and number of fruits per plant exhibited the maximum positive direct effect on the fruit yield. For selecting high yielding genotypes, special importance should be given on fruit weight, fruit length, fruit diameter and number of fruits per plant. This is in agreements with the findings of Kumar $e t$ al., (2007) in bottle gourd, Rao et al., (2000); Ananthan and Krishnamoorthy 2017 in ridge gourd. In the present study the traits fruit weight, fruit length, fruit diameter and number of fruits were more contributions to increase the yield. The residual effects of path coefficient were found to be low in two crosses indicated that most of the traits have respectable correlation with yield. These results are in conformity with Dey et al., (2009) in bitter gourd and Husna et al., (2011) in bottle gourd.

\section{References}

Al-Jibouri, H.H., Miller, P.A. and Robinson, H.F. 1958. "Genotypic and environmental variances and covariances in upland cotton crosses of interspecific origin". Agron. J., 50: 633-37.

Ananthan, M, and V Krishnamoorthy. 2017. "Genetic Variability, Correlation and Path Analysis in Ridge Gourd (Luffa acutangula (Roxb) L." International Journal of Current Microbiology and Applied Sciences, 6 (6):3022-3026.

Choudhary, BR, and SureshKumar. 2011. "Genetic analysis in ridgegourd [Luffa acutangula (Roxb.) L.] under hot arid conditions." Indian journal of arid Horticulture, 6 (1-2): 55-58.

Devi, N Deepa, and S Mariappan. 2013.
"Genetic variability, heritability and genetic advance for yield and its components snake gourd (Trichosanthes anguina L.)." African Journal of Agricultural Research, 8 (28):3857-3859.

Dey, SS, TK Behera, AD Munshi, and R Bhatia. 2009. "Genetic variability, genetic advance and heritability in bitter gourd (Momordica charantia L.)." Indian Agriculturist, 53 (1/2):712.

Husna, A, F Mahmud, MR Islam, MAA Mahmud, and M Ratna. 2011. "Genetic variability, correlation and path co-efficient analysis in bottle gourd (Lagenaria siceraria L.)." Advances in Biological Research, 5 (6):323-327.

Kanimozhi, R, G Mohammed Yassin, S Ramesh Kumar, V Kanthaswamy, and S. Thirumeni. 2015. "Genetic Analysis in Segregating Generation of Wax Gourd." International Journal of Vegetable Science, 21 (3):281-296.

Karthik, D, B Varalakshmi, G Kumar, and N Lakshmipathi. $2017 . \quad$ "Genetic variability studies of ridge gourd advanced inbred lines (Luffa acutangula (L.) Roxb.). International Journal of Pure \& Applied Bioscience, 5 (6):1223-1228.

Koppad, SB, ML Chavan, RH Hallur, V Rathod, and T Shantappa. 2015. "Variability and character association studies in ridge gourd (Luffa acutangula Roxb.) with reference to yield attributes." Journal of Global Biosciences, 4 (5): 2332-2342.

Kumar, Sanjay, R Singh, and AK Pal. 2007. "Genetic variability, heritability, genetic advance, correlation coefficient and path analysis in bottle gourd." Indian Journal of Horticulture, 64(2): 163-168.

Lakshmi, M, LK Haribabu, and GLK Reddy. 
2000. "Character association and path coefficient studies in pumpkin (Cucurbita moschata Duch ex. Poir)." The Andhra Agric J., 49:80-85.

Narasannavar, AR, VD Gasti, T Shantappa, R Mulge, TB Allolli, and N Thammaiah. 2014. "Heterosis studies in ridge gourd [Luffa acutangula (L.) Roxb.]." Karnataka J. Agric. Sci., 27 (1):47-51.

Puddan, M. 2000. "Genetic variability studies in F2 and F3 generation of bitter gourd (Momordica charantia L.)." M.Sc. (Hort.) Thesis, Agricultural College and Research Institute, Madurai.

Rahman, AHMM, M Anisuzzaman, Ferdous Ahmed, AKMR Islam, and ATM Naderuzzaman. 2008. "Study of nutritive value and medicinal uses of cultivated cucurbits." Journal of Applied Science Research, 4 (5): 555558.

Rahman, MA, MD Hossain, MS Islam, DK Biswas, and M Ahiduzzaman. 2002. "Genetic variability, heritability and path analysis in snake gourd (Trichosanthes anguina L.)." Pakistan Journal of Biological Sciences, 5 (3):284-286.

Rajawat, KS, and JP Collis. 2017. "Genetic variability, heritability and genetic advances analysis for quantitative and qualitative traits in Cucumber (Cucumis sativus L.)." Journal of Pharmacognosy and Phytochemistry, 6(4): 882-885.

Rao, N, B Rao, and I Reddy. 2000. "Character association and path correlation studies in ridge gourd [Luffa acutangula $\mathrm{L}$. Roxb.]." The Andhra Agricultural Journal, 47 (1-2):103-107.

Sahitya, G. 2001. "Genetic evaluation of F2 and F3 generations in snake gourd (Trichosanthes anguina L.)." M.Sc. (Hort.). Thesis, Agricultural College and Research Institute, Madurai.

Samadia, DK. 2011. "Genetic variability studies in ridge gourd under arid environment." Indian Journal of Horticulture, 68(2): 275-277.

Sampath, S and V Krishnamoorthy. 2017. "Genetic Variability, Correlation and Path Analysis in Pumpkin (Cucurbita moschata Duch. ex. Poir)." Int. J. Curr. Microbiol. App. Sci., 6 (6):3027-3035.

Tamilselvi, N. 2010. "Studies on heterosis and combining ability in pumpkin (Cucurbita moschata Duch. ex Poir)." M.Sc. (Hort.) Thesis, Tamil Nadu Agricultural University, Coimbatore, India.

\section{How to cite this article:}

Kannan, A. and Rajamanickam, C. 2019. Genetic Variability, Correlation and Path Analysis of F5 Generation of Ridge Gourd (Luffa acutangula (L.) Roxb.) for Yield and Quality. Int.J.Curr.Microbiol.App.Sci. 8(11): 1153-1164. doi: https://doi.org/10.20546/ijcmas.2019.811.136 